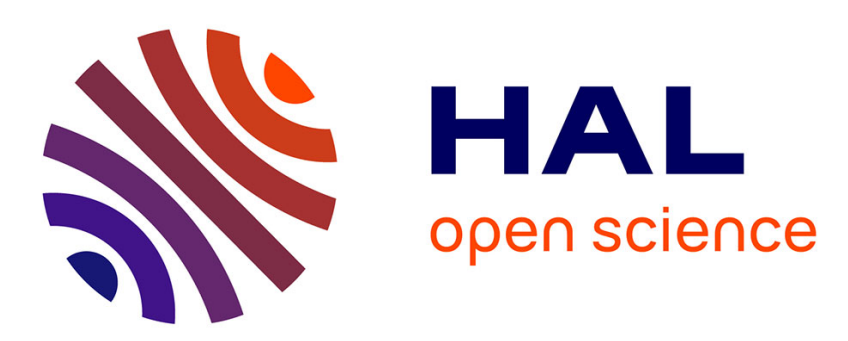

\title{
Fabrication of ultrathin artificial basement membrane for epithelial cell culture
}

Elrade Rofaani, Juan Peng, Li Wang, Yong He, Boxin Huang, Yong Chen

\section{To cite this version:}

Elrade Rofaani, Juan Peng, Li Wang, Yong He, Boxin Huang, et al.. Fabrication of ultrathin artificial basement membrane for epithelial cell culture. Microelectronic Engineering, 2020, 232, pp.111407. 10.1016/j.mee.2020.111407 . hal-03013350

\section{HAL Id: hal-03013350 https://hal.science/hal-03013350}

Submitted on 18 Nov 2020

HAL is a multi-disciplinary open access archive for the deposit and dissemination of scientific research documents, whether they are published or not. The documents may come from teaching and research institutions in France or abroad, or from public or private research centers.
L'archive ouverte pluridisciplinaire HAL, est destinée au dépôt et à la diffusion de documents scientifiques de niveau recherche, publiés ou non, émanant des établissements d'enseignement et de recherche français ou étrangers, des laboratoires publics ou privés. 


\title{
Fabrication of ultrathin artificial basement membrane for epithelial cell culture
}

\author{
Elrade Rofaani ${ }^{\mathrm{a}, \mathrm{b}}$, Juan Penga ${ }^{\mathrm{a}}$, Li Wang ${ }^{\mathrm{c}}$, Yong He ${ }^{\mathrm{a}}$, Boxin Huang ${ }^{\mathrm{a}}$ and Yong Chen ${ }^{\mathrm{a}^{*}}$ \\ a PASTEUR, Département de chimie, École normale supérieure, PSL University, \\ Sorbonne Université, CNRS, 75005 Paris, France \\ ${ }^{\mathrm{b}}$ The Agency for Assessment and Application of Technology, 10340 Jakarta, Indonesia \\ ${ }^{\mathrm{c}}$ MesoBioTech, 231 Rue Saint-Honoré, 75001 Paris, France \\ e-mail: yong.chen@ens.psl.eu
}

\begin{abstract}
Basement membranes are essential for epithelial and endothelial tissue organization. To mimic them, we developed a fabrication method to produce ultrathin membrane of collagen IV and laminin. A honeycomb microframe of thickness $50 \mu \mathrm{m}$ and compartment-size $400 \mu \mathrm{m}$ was firstly patterned in polyethylene glycol diacrylate (PEGDA) by using lithography and vacuum assisted UV curing techniques. Then, a monolayer of gelatin nanofibers was electrospun and crosslinked on the microframe to form a secondary structure, i.e. a fiber mesh with much smaller pore sizes, in each of the honeycomb compartments. Finally, a collagen IV-laminin gel layer was deposited and dehydrated, leading to the formation of an ultrathin membrane over a large area with a nanofiber backbone. Such an artificial basement membrane is mechanically stable and fully biocompatible. It is also semi-permeable which slowed down considerably the diffusion of large size molecules. More importantly, it could be used to improve the monolayer formation of epithelial cells. Thus, this culture device recapitulates the biological basement membrane, permits the epithelial tissue formation, and allows mimicking a more sophisticated cellular microenvironment.
\end{abstract}

Keywords: Basement membrane, monolayer nanofibers, epithelial culture 


\section{Introduction}

Basement membranes (BMs) are sheet-like extracellular matrix (ECM) on which epithelial or endothelial cells stay and organize [1-7]. These sheet-like structures are generally made of collagen IV, laminin, nidogen, and heparan sulfate proteoglycans with a thickness less than $100 \mathrm{~nm}$. While collagen IV and laminin form two independent polymeric networks, other BM proteins make them associated and stable. BMs promote the formation of epithelial and endothelial layers via specific cell adhesion molecules and sulfated glycolipids, which are a priori organ and tissue dependent but share the common ground of being ultrathin and filtration barrier with selective molecular permeability. Although the importance of BMs has long been recognized, only a limited number of investigations have been reported to mimic such a structure [8-10]. Hozumi et al. conjugated laminin-derived peptide to chitosan matrices to mimic the basement membrane but the produced peptide-chitosan was only used for surface treatment of the culture plates [8]. Rossi et al. used electrospinning technique to produce polyester meshes with specific peptide sequences, which allowed co-culture of skin cells [9]. Similarly, Nishiguchi et al. used a mixture of polyester and polyethylene glycols to produce electrospun nanofibers for a bipolar cultured alveolar-capillary barrier model [10]. More generally, natural ECM proteins and synthetic polymers could be used to produce 2D and 3D matrices for cell culture studies but most of the proposed structures did not take into account the sheet-like morphology of nature BMs [11-13]. In particular, the plastic or elastomer membranes widely used are inherently inappropriate for BM mimicking since they are with large through holes, not thin enough, and cannot be homogeneous for the supported cell layers [14-17]. In this regard, a sheet-like scaffold made of continuous collagen IV-laminin ultrathin film is highly desired for most of the BM functions.

Here, we report on a fabrication strategy to achieve such an artificial basement membrane $(\mathrm{ABM})$. We firstly patterned a polymer honeycomb frame of $50 \mu \mathrm{m}$ thickness and $400 \mu \mathrm{m}$ holesizes and then electrospun on it a monolayer of gelatin nanofibers with pores of much smaller sizes $[18,19]$. After crosslinking and different from our previous studies, a solution of collagen IV and laminin mixture was pipetted on the frame-nanofiber assembly. After dehydration, a stable collagen IV-laminin membrane of thickness in the order of $100 \mathrm{~nm}$ could be obtained with a porous fiber backbone. The formation of such ultrathin membranes of large surface should be difficult, and we believe that this hierarchical structure is relevant and applicable to BM related studies. For simplicity, only two major components, i.e., collagen IV and laminin, were used, without taking into account the composition of specific tissue or organ. We will describe the fabrication detail and show the preliminary characterization results of the proposed ABMs, which clearly demonstrated the advantage of using ABMs for the monolayer formation of epithelial cells. 


\section{Materials and methods}

\subsection{Fabrication of ABMs}

We followed the same fabrication process of our previous work to obtain a patch form of culture device made of honeycomb microframe and monolayer of gelatin nanofibers $[18,19]$. In addition, we deposited a gel solution of collagen IV and laminin mixture on the frame-fiber system, which became ultrathin membrane after dehydration (Fig. 1). Briefly, a honeycomb structure of 400- $\mu \mathrm{m}$ hole size, $50-\mu \mathrm{m}$ linewidth and 50- $\mu \mathrm{m}$ thickness was defined in a SU-8 photoresist (Microchem, France) by photolithography. This pattern was sequentially replicated into polydimethylsiloxane (PDMS, Eleco-EFD, France) by casting and then from PDMS to polyethylene glycol diacrylate (PEGDA) by vacuum assisted UV-curing. Afterwards, the frame replica was coated by $10 \mathrm{~nm} \mathrm{Au}$ and gelatin nanofibers were electrospun. Here, 10wt\% gelatin (\#61890, Sigma-Aldrich, France) was dissolved in a mixed solution of acetic acid, ethyl acetate (\#270989, Sigma-Aldrich, France) and DI water at a volume ratio of 21:14:10. The nanofibers were deposited at distance $10 \mathrm{~cm}$ and voltage $11 \mathrm{kV}$ for 3 and $5 \mathrm{~min}$, controlled with a high voltage supply (Heinzinger, Germany), a syringe-pump (\#78-9100B, Kd Scientific, USA) at a feeding rate of $0.2 \mathrm{ml} / \mathrm{h}$. After electrospinning, gelatin nanofibers were dried in a desiccator overnight and cross-linked in a 0.2 M mixture of 1-ethyl-3-(3-dimethylaminopropyl) carbodiimide hydrochloride (EDC) (\#6383, Sigma-Aldrich, France) and N-hydroxysuccinimide (NHS) (\#130672, Sigma-Aldrich, France) in ethanol for $4 \mathrm{~h}$. After crosslinking, samples were rinsed with $99.5 \%$ ethanol three times and dried in vacuum overnight to remove the remaining chemicals.

The above fabricated frame-nanofiber system was used as support for the final deposition of ABM materials. A $50 \mu 1$ solution composed of $0.05 \%$ or $0.1 \%(w / v)$ collagen IV (\#C7521, SigmaAldrich, France) in water containing $0.1 \mathrm{M}$ acetic acid and 2\% (v/v) laminin (\#L2020, SigmaAldrich, France) was pipetted on the frame-nanofiber system. After dehydration at $37^{\circ} \mathrm{C}$ for $3-5 \mathrm{~h}$, a continuous film of collagen IV-laminin mixture could be obtained and its morphology analysis was performed with a tabletop scanning electron microscopy (SEM, TM-3030, Hitachi, Japan).

\subsection{Permeability test}

Molecular diffusion across an ABM was studied using $70 \mathrm{kDa}$ Rhodamine B isothiocyanatedextran (RITC) (\#R9379, Sigma-Aldrich, France) at a concentration of $250 \mu \mathrm{g} / \mathrm{mL}$ in a mixture of 50\% Minimum Essential Medium (MEM) (\#10370-021, Gibco, France) and 50\% Dulbecco's Phosphate Buffer Saline (DPBS) (\#14190-094, Gibco, France). For comparison, measurements were performed with a patch without gel layer deposition and a Geltrex (\#1413202, Gibco, France) coated patch. They were all mounted in a homemade transwell support (Boyden chamber) and placed in a 6 well plate (\#734-2323, VWR, France). $0.5 \mathrm{~mL}$ buffer with RITC and $2.0 \mathrm{~mL}$ buffer 
without RITC were injected separated in the apical and basal sides of the chamber. After diffusion for $24 \mathrm{~h}, 300 \mu \mathrm{L}$ solution were taken from the basal side of each well. $100 \mu \mathrm{L}$ of the solution was placed in a 96 well plate (\#353072, Falcon-Corning, USA) in triplicate and the fluorescence intensity of the samples was measured at $\lambda_{\text {exc }}: 530 / 25 \mathrm{~nm}$ and $\lambda_{\text {ems }}: 645 / 40 \mathrm{~nm}$ using fluorescence plate reader (Synergy HT, BioTek with Gen $^{\mathrm{TM}}$ software). Then, the percentage of diffused molecules was deduced by

$$
\text { Diffusion }(\%)=\frac{I V_{\text {apical }}}{I_{0} V_{\text {basal }}} \times 100 \%
$$

where $I_{0}(I)$ and $V_{\text {apical }}\left(V_{\text {basal }}\right)$ are the fluorescence intensity (arbitrary unit) and the volume of the initial (final) solution in apical (basal) side of the membrane.

\subsection{Cell seeding and culture}

Before cell seeding, ABMs were sterilized in ethanol for 15 min then washed in DPBS, dried under ultraviolet light for 30 min. Alveolar epithelial type II A549 cells (\#86012804, SigmaAldrich, France) were cultured in DMEM/F-12 with glutamax supplement (\#10565018, Gibco, France) containing 10\% fetal bovine serum (\#16000044, FBS, Gibco, France) and 1\% penicillin/streptomycin (\#15140122, Gibco, France) at $37{ }^{\circ} \mathrm{C}$ with $5 \% \mathrm{CO}_{2}$. The Boyden chamber inserted with patch was used as air-liquid interface cell culture model and placed in 6 well plate. Firstly, cells were seeded in the top compartment of Boyden chamber at a density of $2 \times 10^{5}$ cells per patch and incubated for 3 days. Then, the culture medium was replaced by a base medium of DMEM/F-12 with glutamax supplement containing 4\% FBS, $1 \%$ penicillin/streptomycin, and 250 nM dexamethasone (\#D4902, Sigma-Aldrich, France). After one day culture, the medium in the top compartment was removed, leaving the apical surface of the cells exposed to air (in an atmosphere of $5 \% \mathrm{CO}_{2}$ at $37^{\circ} \mathrm{C}$ ). Afterwards, the culture was continued and the medium in the low compartment was changed every two days for up to 17 days. Here, the culture medium with a lower FBS concentration and $250 \mathrm{nM}$ dexamethasone was used for long-term culture.

\subsection{Immunostaining observation}

Cells were rinsed with DPBS and fixed with 4\% paraformaldehyde (\#P6148, Sigma-Aldrich, France) for $15 \mathrm{~min}$. Samples were permeabilized with 0.5\% Triton X-100 (\#HFH10, Thermofisher, France) for 10 min and saturated with DPBS supplemented with $0.1 \%$ Triton X-100 and 3\% BSA (\#A9056, Sigma-Aldrich, France) for $2 \mathrm{~h}$ at room temperature. Afterwards, staining was performed with primary antibodies for overnight at $4{ }^{\circ} \mathrm{C}$, which followed by incubation with fluorescent second antibodies for $2 \mathrm{~h}$. The primary antibody Alexa fluor 488-conjugated anti-collagen IV (\#539871-82, Invitrogen, France) and anti-laminin (\#PA1-16730, Invitrogen, France) were used for staining ABM, while unconjugated antibody ZO-1 (\#40-2200, Invitrogen, France) and E-cadherin (\#14-3249-80, Invitrogen, France) were followed by the secondary antibody Alexa Fluor 488 and 
647 (\#A-11008 and \#A-31573, Invitrogen, France) were used for staining of A549 cells. Finally, nucleus was stained with DAPI (\#62247, Invitrogen, France) for $30 \mathrm{~min}$ at room temperature. Samples were observed with a confocal microscope (LSM 710, Zeiss, France). Images were collected as TIF files and analyzed with software ImageJ.

\section{Results and Discussion}

In general, an ultrathin membrane with uniform biochemical properties is physiologically more relevant than thicker membranes with through holes for epithelial and endothelial cell culture. However, it is hardly conceivable to produce a bio-membrane of thickness in the order of $100 \mathrm{~nm}$ over a large area $\left(\mathrm{a}\right.$ few $\mathrm{mm}^{2}$ ). To overcome this difficulty, we adopted a hierarchic device design by embedding a nanofiber backbone inside the membrane and using a honeycomb microframe as carrier of an ultrathin membrane. We firstly created a large pore-sized PEGDA microframe. Then, we deposited on it a monolayer of crosslinked gelatin nanofibers with a high porosity. Typically, the fiber diameter is in the range of 100 to $500 \mathrm{~nm}$ [18], the pore size is in the order of a few $\mu \mathrm{m}$, and the contact angle of the fiber layer is about $45^{\circ}$. With such a mesh structure, thin gel layers of collagen IV-laminin can be easily formed in the porous areas of the fibers. After dehydration, the thickness of the gel layer became smaller and the fiber-gel assembly was self-organized, giving rise to an ultrathin membrane with a fiber mesh backbone. Since this membrane is made of BM specific proteins, it is naturally bio-compatible. As expected, the fabricated membrane is mechanically stable and easy to use. Also, the developed fabrication method is straightforward and applicable to large scale manufacturing.

Figure 2 shows scanning electron microscope (SEM) images of fabricated membranes. As expected, the monolayer of nanofibers looked like a backbone to support the ultrathin membrane in the open areas of PEGDA microframe (Fig. 2A). From these SEM images, the pore sizes of the fibers can be determined. Statistically, the area of the pores varied in the range of 5 to $50(10) \mu \mathrm{m}^{2}$ for the fibers produced by 3 (5) min electrospinning, corresponding a porosity of $62 \%(50 \%)$ respectively. With a gel of $0.1 \%$ collagen IV-laminin, the thickness of membrane after dehydration can be as small as $166.5 \pm 35.5 \mathrm{~nm}$ (Fig. $2 \mathrm{C}, \mathrm{n}=3$ ). Note that with the same gel concentration, such an ultrathin membrane can be more easily broken in large pores obtained by 3 min electrospinning (Fig. 2B). Decreasing the pore size by, for example, 5 min electrospinning resulted in a more stable membrane. Similarly, a smaller gel concentration (from $0.1 \%$ to $0.05 \%$ ) did not allow the formation of a continuous thin membrane (Fig. 2F). Clearly, the thickness of the membrane is gel concentration dependent and $0.1 \%$ gel concentration results in the most successful ABM for 5 min electrospun nanofibers. 
. To elucidate the tradeoff between the pore size and the membrane thickness, we consider a gel layer embedded in a pore of radius $R$ and thickness $h$. The surface energy of the gel layer $E_{s}=$ $2 \gamma \pi R^{2}$, the contact energy of the gel anchoring the mesh $E_{a}=2 \gamma^{\prime} \pi h R$, where $\gamma$ and $\gamma^{\prime}$ are respectively the energy density of air-gel and gel-solid. For the formation of a stable gel layer, $E_{s}<E_{a}$, i.e., $R<\left(\frac{\gamma^{\prime}}{\gamma}\right) h$. This means that the pore size has to be limited in order to have a small membrane thickness.

To evaluate the quality of the deposition of the collagen and laminin, immunofluorescence images were taken after anti-collagen (green) and anti-laminin (red) staining (Fig. 3). As expected, on the contrary to the patch without gel deposition, the fabricated ABMs showed both homogenous green and red fluorescence over the whole active areas, indicating excellent coverage of the collagen IV and laminin in the pore areas.

The fabricated ABMs have also been used for permeability test. Fig. 4 shows the percentage of diffusion of molecule RITC $70 \mathrm{kDa}$ across a patch without gel treatment, an ABM and a Geltrex coated patch for $24 \mathrm{~h}$. As can be seen, the patch was highly permeable to RITC $70 \mathrm{kDa}$ molecules because of the high porosity of the monolayer nanofibers. In contrast, only about $20 \%$ of RITC 70 $\mathrm{kDa}$ molecules could be diffused through ABM and Geltrex coated patches, suggesting a limited permeability of the membrane.

To demonstrate the feasibility of monolayer formation of epithelial cells, A549 cells were cultured on ABMs. It is known that the primary function of BMs is to hold epithelial cells through substrate adhesion molecules and to promote the formation of epithelial layer with junctional proteins like tight junction (TJ) proteins. Both BM and TJ are necessary to maintain cell layer integrity and both play roles of protective and functional barriers. More specifically, continous tight junctions are necessary as a boundary between the apical and basolateral cell surface domains to regulate molecule diffusion along the paracellular pathway [20]. Our results showed that A549 cells on both ABM and patch exhibited continuous ZO-1 and E-cadherin expressions at the cell borders for more than two weeks and that the expression level of ZO-1 and E-cadherin proteins increased with time (Fig.5). However, the expression of ZO-1 and E-cadherin of the cells on ABM was more pronounced and more homogenous than on patch. Here, ZO-1 is one of tight junctionassociated proteins, located at the plasma membrane in the apical junctional region, while Ecadherin is a cell-cell adhesion molecule, located on the lateral plasma membrane slightly below the tight junction. Therefore, our results suggest that ABM enhances the formation of tight junctions. Clearly, at day $9 \mathrm{ZO}-1$ and E-cadherin of the cells on ABM appeared more clearly than on patch. At day 17, they formed a more complete network on ABM than on patch. Finally, the vertical section $(X Z)$ images clearly show a polarity of cells on ABM, which could not be observed with other types of BM mimics [17] and further, suggests the advantage of ABM. This can also be 
seen from the vertical section $(X Z)$ image of cells on patch at day 17, where two cell layers appeared due to absence of BM proteins. A549 cells were derived from lung cancer and are often used as models of alveolar Type II pulmonary epithelium. Our results showed that monolayer of A549 cells could be achieved and sustained on ABM but not on patch. This means that ABM, like natural BM, plays important roles to the homeostasis of epithelial layer by dynamic regulation of cell-BM interaction, cell migration and cell division through a variety of signaling and cytoskeleton regulation events. More investigations are expected to understand these events with ABMs.

Previously, culture patches without collagen IV-laminin treatment could be used as substrates for differentiation of human induced pluripotent stem cells toward cardiomyocytes [18] and neurons [21] as well as primary neuron cells [22] and fibroblasts [23]. In such cases, collagen IVlaminin treatment would not be necessary since these cells were not epithelial. In general, the use of $\mathrm{ABM}$ as substrate should be relevant for epithelial and endothelial culture and tissue modeling where cell polarity and TJs are primarily important.

\section{Conclusion}

We proposed a three-level fabrication strategy to mimic natural BMs. The first-level structure, i.e., the honeycomb microframe, is handleable and compatible to conventional culture. The second-level structure, i.e., the monolayer of crosslinked gelatin nanofibers, serves as backbone to handle an ultrathin membrane. Finally, the ultrathin membrane made of collagen IV-laminin mixture acts as functional ABM. Altogether, the hierarchic structure is mechanically stable and easy to use. Our results showed that $0.1 \%$ gel concentration of collagen IV-laminin mixture was appropriate to form ultrathin films of thickness in the order of $100 \mathrm{~nm}$ in the porous areas of electrospun nanofibers. In addition, such a fabrication strategy is straightforward for the formation of ultrathin membrane with other materials. The permeability of the membrane has been studied with $70 \mathrm{kDa}$ RITC, showing characteristic diffusion behavior. The fabricated membrane has also been used to improve the monolayer formation of epithelial cells. We believe that such an ultrathin ABM should be more appropriate for tissue and organ-on-a-chip studies comparing to the commonly used plastic or elastomeric membranes.

\section{Aknowledgment}

This work was supported by the Agence Nationale de la Recherche under contract No ANR17-CE09-0017 (AlveolusMimics), DIM ELICIT program of Ile-de-France, and PSL Valorization through Pre-maturation project. ER was granted by Indonesia Endowment Fund for Education, Ministry of Finance of The Republic of Indonesia (LPDP). 


\section{References}

[1] G. W. Laurie and C. P. Leblond, "What is known of the production of basement membrane components," J. Histochem. Cytochem., vol. 31, no. 1 A, pp. 159-163, 1983, doi: 10.1177/31.1A_SUPPL.6186721.

[2] M. Paulson, "Basement membrane proteins: Structure, assembly, and cellular interactions," Crit. Rev. Biochem. Mol. Biol., vol. 27, no. 1-2, pp. 93-127, 1992, doi: $10.3109 / 10409239209082560$.

[3] V. S. LeBleu, B. MacDonald, and R. Kalluri, "Structure and function of basement membranes," Exp. Biol. Med., vol. 232, no. 9, pp. 1121-1129, 2007, doi: 10.3181/0703MR-72.

[4] P. D. Yurchenco, "Basement membranes: Cell scaffoldings and signaling platforms," Cold Spring Harb. Perspect. Biol., vol. 3, no. 2, pp. 1-27, 2011, doi: 10.1101/cshperspect.a004911.

[5] A. Pozzi, P. D. Yurchenco, and R. V. Iozzo, "The nature and biology of basement membranes," Matrix Biol., vol. 57-58, pp. 1-11, 2017, doi: 10.1016/j.matbio.2016.12.009.

[6] R. P. Mecham, Ed., Cell biology of extracellular matrix, vol. 69, no. 6. Springer US, 1992.

[7] M. S. Thomsen, L. J. Routhe, and T. Moos, "The vascular basement membrane in the healthy and pathological brain,” J. Cereb. Blood Flow Metab., vol. 37, no. 10, pp. 33003317, 2017, doi: 10.1177/0271678X17722436.

[8] K. Hozumi, J. Kumai, Y. Yamada, and M. Nomizu, "Active peptide-conjugated chitosan matrices as an artificial basement membrane," Polymers (Basel)., vol. 7, no. 2, pp. 281297, 2015, doi: 10.3390/polym7020281.

[9] A. Rossi, L. Wistlich, K. H. Heffels, H. Walles, and J. Groll, "Isotropic Versus Bipolar Functionalized Biomimetic Artificial Basement Membranes and Their Evaluation in LongTerm Human Cell Co-Culture," Adv. Healthc. Mater., vol. 5, no. 15, pp. 1939-1948, 2016, doi: 10.1002/adhm.201600224.

[10] A. Nishiguchi, S. Singh, M. Wessling, C. J. Kirkpatrick, and M. Möller, "Basement Membrane Mimics of Biofunctionalized Nanofibers for a Bipolar-Cultured Human Primary Alveolar-Capillary Barrier Model,” Biomacromolecules, vol. 18, no. 3, pp. 719-727, 2017, doi: 10.1021/acs.biomac.6b01509.

[11] E. S. Place, N. D. Evans, and M. M. Stevens, "Complexity in biomaterials for tissue engineering," Nat. Mater., vol. 8, no. 6, pp. 457-470, 2009, doi: 10.1038/nmat2441.

[12] F. J. O’Brien, "Biomaterials \& scaffolds for tissue engineering," Mater. Today, vol. 14, no. 3, pp. 88-95, 2011, doi: 10.1016/S1369-7021(11)70058-X.

[13] R. Cruz-Acuña and A. J. García, "Synthetic hydrogels mimicking basement membrane 
matrices to promote cell-matrix interactions," Matrix Biol., vol. 57-58, pp. 324-333, 2017, doi: 10.1016/j.matbio.2016.06.002.

[14] D. Huh, B. D. Matthews, A. Mammoto, M. Montoya-Zavala, H. Yuan Hsin, and D. E. Ingber, "Reconstituting organ-level lung functions on a chip," Science (80-. )., vol. 328, no. 5986, pp. 1662-1668, 2010, doi: 10.1126/science.1188302.

[15] A. O. Stucki et al., "A lung-on-a-chip array with an integrated bio-inspired respiration mechanism," Lab Chip, vol. 15, no. 5, pp. 1302-1310, 2015, doi: 10.1039/c4lc01252f.

[16] K. H. Benam et al., "Small airway-on-a-chip enables analysis of human lung inflammation and drug responses in vitro," Nat. Methods, vol. 13, no. 2, pp. 151-157, 2016, doi: 10.1038/nmeth.3697.

[17] N. Higuita-Castro et al., "Using a Novel Microfabricated Model of the Alveolar-Capillary Barrier to Investigate the Effect of Matrix Structure on Atelectrauma," Sci. Rep., vol. 7, no. 1, pp. 1-13, 2017, doi: 10.1038/s41598-017-12044-9.

[18] Y. Tang et al., "Induction and differentiation of human induced pluripotent stem cells into functional cardiomyocytes on a compartmented monolayer of gelatin nanofibers," Nanoscale, vol. 8, no. 30, pp. 14530-14540, 2016, doi: 10.1039/c6nr04545f.

[19] J. S. Y. Chen, Y.D. Tang, L.Wang, “Cell culture device, Patent, WO 2017/005927A, Jan $12, " 2017$.

[20] M. Hirsch and W. Noske, "The tight junction: Structure and function," Micron, vol. 24, no. 3, pp. 325-352, 1993, doi: 10.1016/0968-4328(93)90057-8.

[21] Y. Tang et al., "Effective motor neuron differentiation of hiPSCs on a patch made of crosslinked monolayer gelatin nanofibers," J. Mater. Chem. B, vol. 4, no. 19, pp. 33053312, 2016, doi: 10.1039/c6tb00351f.

[22] Y. Tang, F. P. Ulloa Severino, F. Iseppon, V. Torre, and Y. Chen, "Patch method for culture of primary hippocampal neurons," Microelectron. Eng., vol. 175, pp. 61-66, 2017, doi: 10.1016/j.mee.2017.01.012.

[23] B. Wang et al., "Fabrication of spaced monolayers of electrospun nanofibers for threedimensional cell infiltration and proliferation," Microelectron. Eng., vol. 198, no. March, pp. 73-77, 2018, doi: 10.1016/j.mee.2018.07.005. 


\section{Figure caption :}

Figure 1. Schematic of the fabrication steps of an ultrathin artificial basement membrane (ABM):

(A) Patterning of honeycomb microframe, (B) Electrospinning of monolayer of nanofibers, (C) Deposition of ultrathin film of collagen IV-laminin.

Figure 2. Scanning electron microscope images of the fabricated devices. (A) Overview showing a homogenous gel layer (membrane of collagen IV-laminin) supported by a mesh of monolayer nanofibers (backbone) and honeycomb microframe. (B, C) Detailed view of the sample showing the fibers and the membrane. (D, E) Comparison of two samples obtained by 3 and 5 min nanofiber electrospinning and deposition-dehydration of a gel with $0.1 \%$ collagen IV-laminin. (F) Sample obtained by 5 min electrospinning and deposition-dehydration of a gel of $0.05 \%$ concentration. The red dot squares indicate the zoom areas. Scale bar was shown under each image.

Figure 3. Immunostaining fluorescence images of the membrane structure before (A) and after (B, C) deposition of ultrathin ABM. Immunostaining of collagen IV in green and laminin in red.

Figure 4. Permeability of $70 \mathrm{kDa}$ Rhodamine B isothiocyanate-dextran (RITC) across a membrane for 24 h. Patch: without gel deposition, ABM: patch with a gel layer of collagen IV-laminin, Geltrex: patch after deposition with a commercial gel. Values are means \pm s.e.m. In insert: the homemade transwell-like device used for this experiment.

Figure 5. Confocal immunofluorescence images of A549 cells cultured on a patch and an ultrathin ABM for 9 and 17 days. ZO-1 was stained in green, E-cadherin in red and nucleus in blue. Single confocal sections of monolayer were shown $(X Y)$. Images of vertical sections were presented downside $(X Z)$. The scale bar represents $10 \mu \mathrm{m}$. 
Figure 1

(A) Microframe

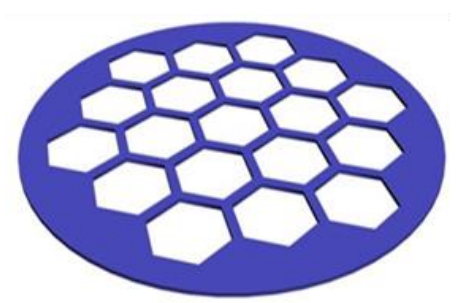

(B) Nanofibers

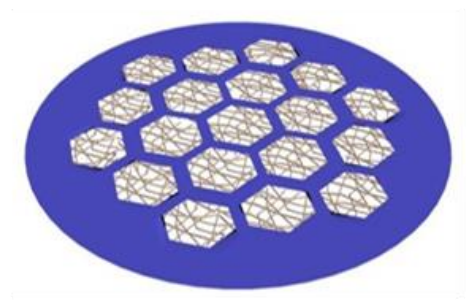

(C) Gel layer

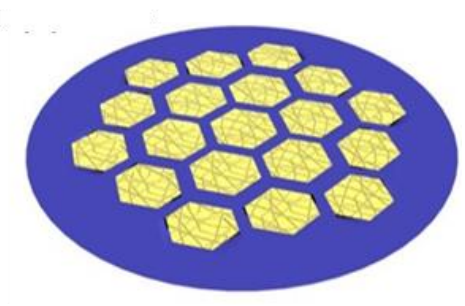

Figure 2
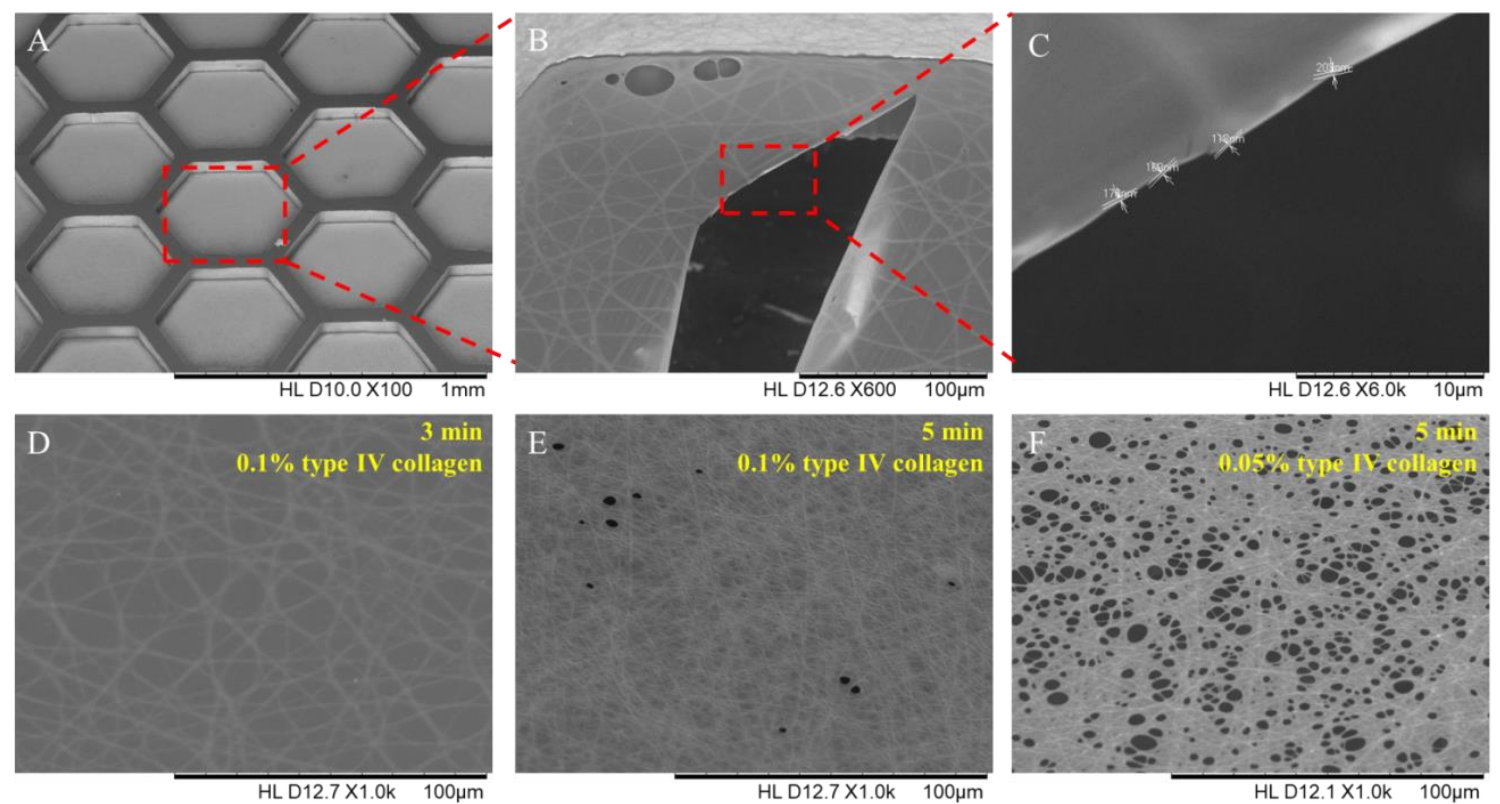
Figure 3
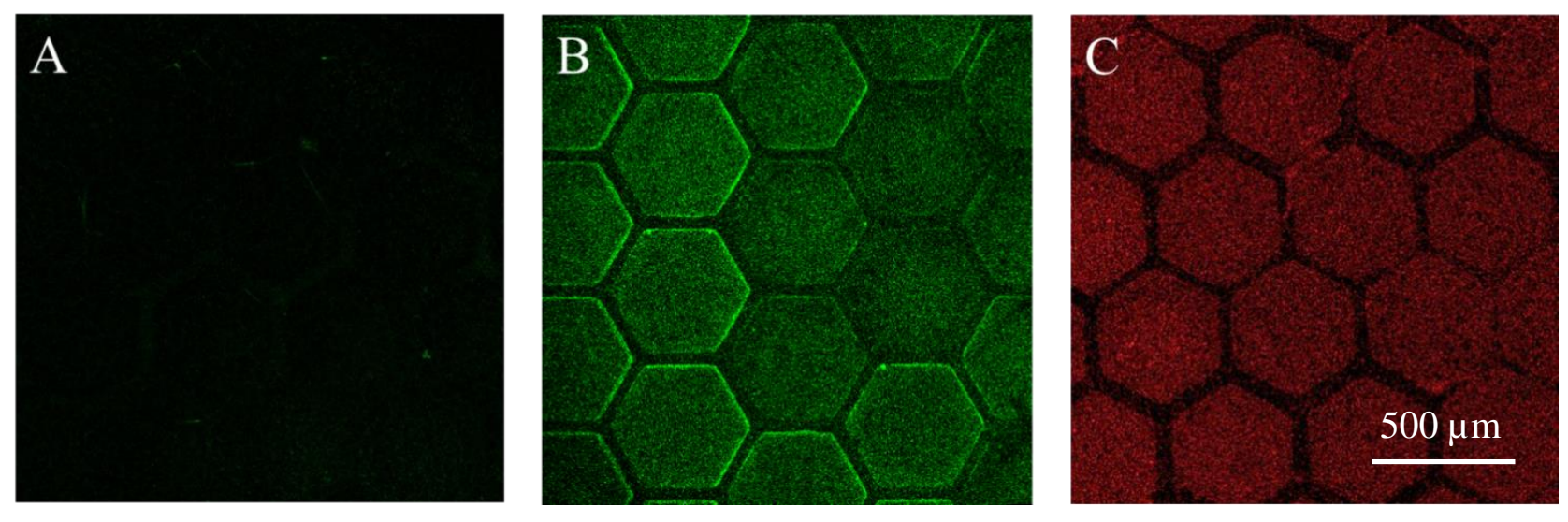

Figure 4

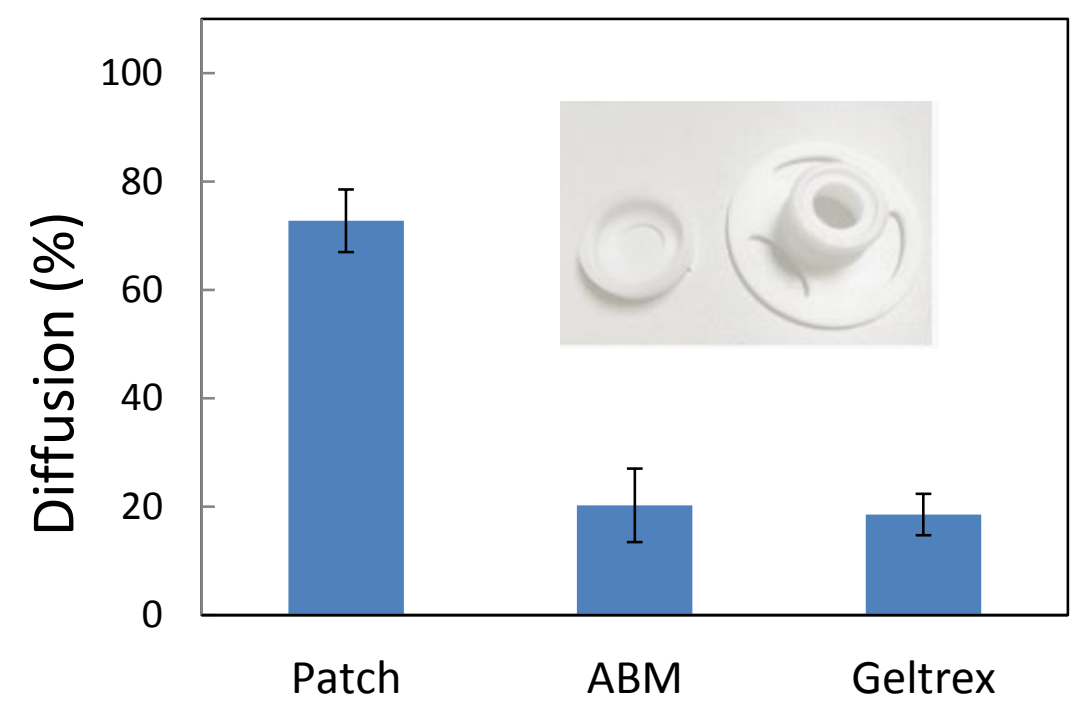


Figure 5

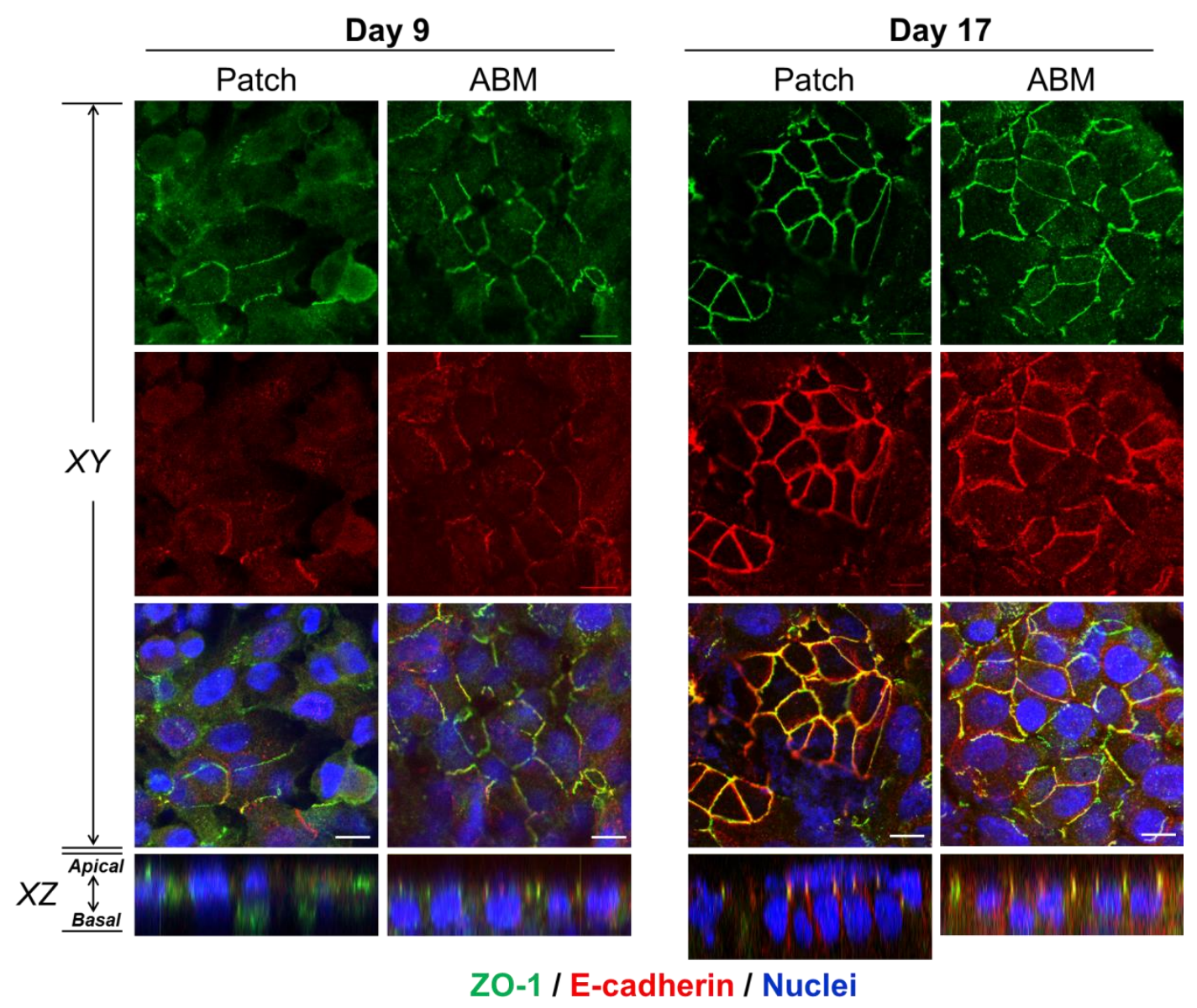

